\title{
Incidence and severity of pertussis hospitalisations in infants aged less than 1 year in 37 hospitals of six EU/ EEA countries, results of PERTINENT sentinel pilot surveillance system, December 2015 to December 2018
}

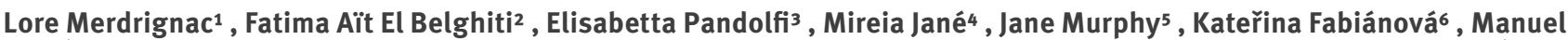

García Cenoz $^{7}$, Elmira Flem ${ }^{8}$, Sophie Guillot ${ }^{9}$, Alberto E Tozzi ${ }^{10}$, Gloria Carmona ${ }^{4}$, Adele Habington ${ }^{11}$, Jana Zavadilová6 ${ }^{6}$, Ana

Navasués ${ }^{12}$, Håkon Bøås ${ }^{8}$, Daniel Lévy-Brühl' ${ }^{2}$, Beatrice Ferrettí ${ }^{3}$, Miguel Lanaspa ${ }^{13}$, Niam O'Sullivan ${ }^{11}$, Pavla Kř́̌žová6, Leticia

Fernandino $^{7}$, Terese Bekkevold ${ }^{8}$, Thomas Hanslik ${ }^{14}$, Carmen Muñoz-Almagro ${ }^{13,15,16}$, Sabrina Bacci ${ }^{17}$, Gianfranco Spiteri ${ }^{17}$, Marta

Valenciano $^{1}$, Alain Moren ${ }^{1}$, PERTINENT Group ${ }^{18}$

1. Epidemiology Department, Epiconcept, Paris, France

2. Direction des maladies infectieuses, Santé Publique France, Paris, France

3. Multifactorial Disease and Complex Phenotype Research Area, Bambino Gesù Children Hospital, Rome, Italy

4. Epidemiological surveillance and response, Public Health Agency of Catalonia, Barcelona, Spain

5. Research, Temple Street Children's University Hospital, Dublin, Ireland

6. National Institute of Public Health, Prague, Czech Republic

7. Instituto de Salud Pública de Navarra, IdiSNA - Navarre Institute for Health Research, Pamplona, Spain

8. Infectious Disease Epidemiology and Modeling, Norwegian Institute of Public Health, Oslo, Norway

9. Biodiversité et Epidémiologie des bactéries et pathogènes, Institut Pasteur, Paris, France

10. Chief Innovation Unit and Clinical Trials, Bambino Gesù Children Hospital, Rome, Italy

11. Microbiology, Our Lady's Children's hospital Crumlin, Dublin, Ireland

12. Clinical Microbiology Service, Complejo Hospitalario de Navarra, Pamplona, Spain

13. Instituto de Recerca Pediatrica Hospital Sant Joan de Deu, Barcelona, Spain

14. Sorbonne University, INSERM, Institut Pierre Louis d’Epidémiologie et de Santé Publique, Paris, France

15. Universitat Internacional de Catalunya, Barcelona, Spain

16. CIBER of Epidemiology and Public Health CIBERESP, Barcelona, Spain

17. European Centre for Diseases Prevention and Control, Stockholm, Sweden

18. The members of the network are listed at the end of the article

Correspondence: Lore Merdrignac (l.merdrignac@epiconcept.fr)

PERTINENT group:

The PERTINENT group is listed at the end of the article.

Citation style for this article:

Merdrignac Lore, Aït El Belghiti Fatima, Pandolfi Elisabetta, Jané Mireia, Murphy Jane, Fabiánová Kateřina, García Cenoz Manuel, Flem Elmira, Guillot Sophie,

Tozzi Alberto E, Carmona Gloria, Habington Adele, Zavadilová Jana, Navasués Ana, Bøås Håkon, Lévy-Brühl Daniel, Ferretti Beatrice, Lanaspa Miguel, O’Sullivan

Niam, Kř́žzová Pavla, Fernandino Leticia, Bekkevold Terese, Hanslik Thomas, Muñoz-Almagro Carmen, Bacci Sabrina, Spiteri Gianfranco, Valenciano Marta, Moren

Alain, PERTINENT Group. Incidence and severity of pertussis hospitalisations in infants aged less than 1 year in 37 hospitals of six EU/EEA countries, results of

PERTINENT sentinel pilot surveillance system, December 2015 to December 2018. Euro Surveill. 2021;26(4):pii=1900762. https://doi.org/10.2807/1560-7917.

ES.2021.26.4.1900762

Introduction: PERTINENT is a pilot active surveillance systaem of infants hospitalised with pertussis in six European Union/European Economic Area countries (37 hospitals, seven sites). Aim: This observational study aimed to estimate annual pertussis incidence per site from 2016 to 2018 and respective trends between 2017 and 2018. Pertussis cases were described, including their severity. Methods: We developed a generic protocol and laboratory guidelines to harmonise practices across sites. Cases were hospitalised infants testing positive for Bordetella pertussis by PCR or culture. Sites collected demographic, clinical, laboratory data, vaccination status, and risk/protective factors. We estimated sites' annual incidences by dividing case numbers by the catchment populations. Results: From December 2015 to December 2018, we identified 469 cases (247 males; 53\%). The median age, birthweight and gestational age were 2.5 months (range: $0-11.6$; interquartile range (IQR):2.5), 3,280 g (range:7004,925; IQR:720) and 39 weeks (range: 25-42; IQR: 2), respectively. Thirty cases (6\%) had atypical presentation either with cough or cyanosis only or with absence of pertussis-like symptoms. Of 330 cases with information, $83(25 \%)$ were admitted to intensive care units including five deceased infants too young to be vaccinated. Incidence rate ratios between 2018 and 2017 were 1.43 in Czech Republic ( $p=0.468), 0.25$ in Catalonia $(p=0.002), 0.71$ in France $(p=0.034), 0.14$ in Ireland $(p=0.002), 0.63$ in Italy $(p=0.053), 0.21$ in Navarra $(p=0.148)$ and zero in Norway. Conclusions: Incidence appeared to decrease between 2017 and 2018 in all but one site. Enhanced surveillance of hospitalised pertussis in Europe is essential to monitor pertussis epidemiology and disease burden.

\section{Introduction}

Most severe cases of pertussis (whooping cough) occur below 5 years of age. Worldwide, it causes substantial mortality in infants $(85,900$ estimated deaths in 2014) [1]. Sixty-three percent of cases aged less than 1 year 


\section{TABLE 1}

Characteristics of PERTINENT study sites, number of cases positive to Bordetella species and annual incidence by study site and year, Europe, 1 December 2015-31 December 2018 ( $\mathrm{n}=41$ sites)

\begin{tabular}{|c|c|c|c|c|c|c|c|c|}
\hline \multicolumn{2}{|c|}{ Study sites } & $\begin{array}{c}\text { Czech } \\
\text { Republic }\end{array}$ & France & Ireland & Italy & Spain, Catalonia & $\begin{array}{l}\text { Spain, } \\
\text { Navarra }\end{array}$ & Norway \\
\hline \multicolumn{9}{|c|}{ Vaccination recommendations: year introduction and doses recommended } \\
\hline \multirow{2}{*}{$\begin{array}{l}\text { Primary } \\
\text { schedule }\end{array}$} & Year of introduction & $2018^{\mathrm{a}}$ & 2013 & 1995 & 1995 & $2016^{\mathrm{b}}$ & $2016^{\mathrm{b}}$ & 1998 \\
\hline & Ages for different doses & $\begin{array}{c}3,5, \\
11-13 \text { months }\end{array}$ & $2,4,11$ months & $\begin{array}{c}2,4 \\
6 \text { months }\end{array}$ & $\begin{array}{l}3,5 \\
11 \text { months }\end{array}$ & $2,4,11$ months & $\begin{array}{c}2,4 \\
11 \text { months }\end{array}$ & $3,5,12$ months \\
\hline \multicolumn{2}{|c|}{ Pregnancy, year of introduction } & 2016 & No & 2013 & 2017 & 2014 & 2015 & No \\
\hline \multicolumn{2}{|c|}{ Cocooning, year of introduction } & No & 2004 & 2013 & No & No & No & No \\
\hline \multicolumn{9}{|c|}{ Participating hospitals and catchment population estimation } \\
\hline \multicolumn{2}{|c|}{$\begin{array}{l}\text { Number of hospitals participating in } \\
\text { PERTINENT }\end{array}$} & 6 & 21 & 2 & 2 & 1 & 4 & $\begin{array}{l}5(2016-2018 / 05) \\
1(\text { from 2018/05) }\end{array}$ \\
\hline \multicolumn{2}{|c|}{$\begin{array}{l}\text { Method used to estimate hospital } \\
\text { catchment population }\end{array}$} & $\begin{array}{l}\text { National } \\
\text { census }\end{array}$ & $\begin{array}{c}\text { National } \\
\text { census } \times \text { estimation of } \\
\text { PERTINENT hospital } \\
\text { coverage [13] }\end{array}$ & $\begin{array}{l}\text { National } \\
\text { census }\end{array}$ & $\begin{array}{l}\text { Regional } \\
\text { census }\end{array}$ & $\begin{array}{c}\text { National } \\
\text { census } \times \text { estimation of } \\
\text { PERTINENT hospital } \\
\text { coverage }\end{array}$ & $\begin{array}{l}\text { National } \\
\text { census }\end{array}$ & $\begin{array}{l}\text { Regional census } \\
\text { prorata temporis }\end{array}$ \\
\hline \multicolumn{2}{|c|}{ Screened infants in PERTINENT } & 73 & 546 & 138 & 509 & 207 & 118 & 523 \\
\hline \multicolumn{2}{|c|}{ Bordetella pertussis cases $^{c}$} & 25 & $199^{\mathrm{d}}$ & 30 & $145^{\mathrm{e}}$ & 50 & 13 & 7 \\
\hline \multicolumn{2}{|c|}{ Bordetella parapertussis cases } & 0 & 1od & 2 & NA & 1 & 4 & NA \\
\hline \multicolumn{2}{|c|}{ Bordetella holmesii cases } & o & 2 & 0 & NA & 1 & 1 & NA \\
\hline \multicolumn{2}{|c|}{ Other Bordetella species ${ }^{f}$} & o & 16 & o & NA & 3 & o & NA \\
\hline \multicolumn{9}{|c|}{ Total Bordetella pertussis cases and incidence, by year of study } \\
\hline \multicolumn{9}{|l|}{2016} \\
\hline \multicolumn{2}{|c|}{ Number of cases 2016} & 8 & 45 & 6 & 61 & 19 & 7 & 4 \\
\hline \multicolumn{2}{|c|}{ Catchment population 2016} & 65,638 & 190,077 & 25,110 & 34,428 & 12,138 & 5,875 & 25,545 \\
\hline \multicolumn{2}{|c|}{ Incidence per 100,000 infants } & 12.2 & $29.1^{\mathrm{g}}$ & $57.2^{\mathrm{g}}$ & $211.9^{g}$ & 156.5 & 119.1 & $17.1^{\mathrm{g}}$ \\
\hline \multicolumn{2}{|c|}{$95 \%$ exact confidence interval } & $(5 \cdot 3-24.0)$ & $(21.2-38.9)$ & $\begin{array}{l}(21.0- \\
124.4)\end{array}$ & $(162.1-272.1)$ & $(94 \cdot 3-244 \cdot 3)$ & $(47.9-245 \cdot 3)$ & $(4.7-43.8)$ \\
\hline \multicolumn{9}{|l|}{2017} \\
\hline \multicolumn{2}{|c|}{ Catchment population 2017} & 68,128 & 185,420 & 23,267 & 33,811 & 12,056 & 5,856 & 25,479 \\
\hline \multicolumn{2}{|c|}{ Incidence per 100,000 infants } & 10.3 & 49.1 & 90.3 & 139.0 & 207.4 & 85.4 & 11.8 \\
\hline \multicolumn{2}{|c|}{$95 \%$ exact confidence interval } & $(4.1-21.2)$ & $(39.5-60.3)$ & $\begin{array}{l}(55.9- \\
137.9)\end{array}$ & $\begin{array}{c}(102.2- \\
184.8)\end{array}$ & $(134.2-306.0)$ & $(27.7-199.1)$ & $(2.4-34.4)$ \\
\hline \multicolumn{9}{|l|}{2018} \\
\hline \multicolumn{2}{|c|}{ Number of cases 2018} & 10 & 63 & 3 & 28 & 6 & 1 & 0 \\
\hline Catchment & opulation 2018 & 68,061 & 181,481 & 23,191 & 31,953 & 11,593 & 5,708 & $14,308^{\mathrm{h}}$ \\
\hline $\begin{array}{l}\text { Incidence } \\
\text { January } 20\end{array}$ & $\begin{array}{l}\text { r 100,000 infants } \\
\text {-December } 2018\end{array}$ & $14 \cdot 7$ & 34.7 & 12.9 & 87.6 & 51.8 & 17.5 & 0.0 \\
\hline $95 \%$ exact & nfidence interval ${ }^{i}$ & $(7.0-27.0)$ & $(26.7-44.4)$ & $(2.7-37.8)$ & $(58.2-126.6)$ & $(19.0-112.6)$ & $(0.4-97.6)$ & $(0-25.8)$ \\
\hline
\end{tabular}

NA: not available; PERTINENT: Pertussis in Infants European Network.

a Before 2018: doses at 2, 3, 4 and 10 months.

b Before 2016: doses at 2, 4 and 6 months.

c Percentage of positivity among screened infants is expected to vary across sites due to the variation of hospitalisation likelihood of infants with pertussis-like symptoms.

d Including one co-infection of Bordetella pertussis and Bordetella parapertussis.

e Including nine cases recruited in 2015.

${ }^{\mathrm{f}}$ Laboratory could not confirm and differentiate the Bordetella species.

${ }^{\mathrm{g}}$ Incidence calculated prorata temporis based on available data in 2016 due to a progressive implementation of the surveillance.

h Withdrawal of four hospitals.

¡ 'Exact confidence interval' for incidence as the exact binomial confidence interval (i.e. Clopper-Pearson interval) was computed. 
Flowchart of hospitalised infants' (<1 year) inclusion in or exclusion from the PERTINENT study, Europe, 1 December 2015-31 December 2018 ( $n=2,144$ hospitalised infants)

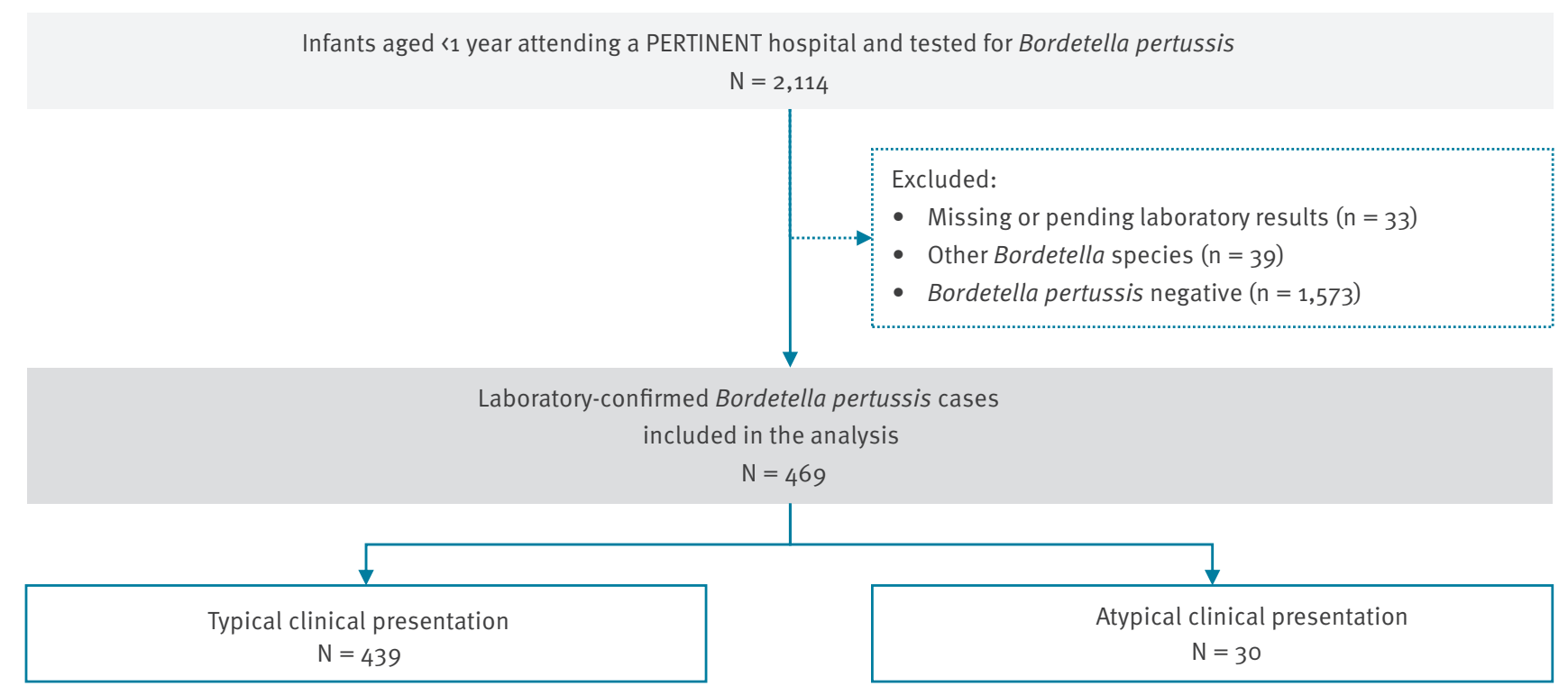

PERTINENT: Pertussis in Infants European Network.

reported to the European Centre for Disease Prevention and Control (ECDC) in 2017 required hospitalisation [2]. Severe complications include pneumonia, seizures, encephalopathy and death.

In neonatal infection, the cough with the characteristic whoop might be absent. The initial finding for Bordetella pertussis infection is frequently apnoea [3]. Since infants and neonates often have a clinical presentation that is atypical or similar to several other respiratory diseases, Vittuci et al. support a routine pertussis laboratory diagnosis in all infants aged less than 3 months with acute respiratory symptoms [4]. Adolescents ( $\geq 11$ years of age) and adults ( $\geq 18$ years of age) can develop a mild, often undiagnosed, form of the disease and represent a reservoir of transmission for infants.

Pertussis is one of the least controlled vaccinepreventable diseases in European Union/European Economic Area (EU/EEA) countries [5]. The number of pertussis cases reported to ECDC increased since 2011 despite a primary series of acellular pertussis vaccine coverage exceeding $90 \%$ in most countries [6]. The last peak incidence year occurred in 2012 with 42,500 reported cases, impacting 19 of 28 countries with different magnitude [5]. It affected adolescents, adults and particularly children too young to be vaccinated or to have completed the primary series. This increase may be explained by improved diagnostic methods, increased disease awareness, waning of acellular-vaccine-induced protection, or a lower vaccine effectiveness (VE) due to bacterial mutation [7].
At EU/EEA level, the routine pertussis surveillance systems are heterogeneous. Under-diagnosis and undernotification also differ across EU/EEA countries [8]. Even though a majority uses the EU case definition $[9,10]$, differences in laboratory procedures, completeness of reporting and differences in disease awareness still remain [8]. Most case definitions do not distinguish between all Bordetella species that can have similar respiratory presentation and include indifferently $B$. pertussis, B. parapertussis, B. holmesii and B. bronchiseptica. Bordetella species may not have the same pathogenicity and may not be all directly targeted by pertussis-containing vaccine [3]. Therefore, existing surveillance systems make comparison of pertussis immunisation strategies and estimates of pertussis incidence at EU/EEA level difficult. Following the 2012 outbreak, an ECDC consultation resulted in a recommendation to conduct studies in the EU/EEA to measure the burden of pertussis including infant hospitalisations [11]. In recent years, some countries have enhanced surveillance of pertussis at hospital [12-14] or population level [15].

In 2015, ECDC initiated PERTINENT, 'Pertussis in Infants European Network', a hospital-based active pilot surveillance system in seven study sites using the same pertussis surveillance protocol. The surveillance system has two main objectives. The first is to identify the trends in incidence of laboratory-confirmed pertussis in hospitalised infants ( $\leq 1$ year old) for the respective study sites. The second is to estimate VE using the test-negative design. In the current study, annual incidences per site are estimated from 2016 to 2018 as well as the change in incidence in 2018 compared 


\section{TABLE 2A}

Hospitalised Bordetella pertussis cases aged $<1$ year by age group, sex, laboratory components, clinical presentation, most likely source of infection and severity criteria, PERTINENT study, Europe, 1 December 2015-31 December 2018 (n=469 cases)

\begin{tabular}{|c|c|c|c|c|c|c|c|c|}
\hline \multirow[t]{2}{*}{ Characteristic } & & \multicolumn{2}{|c|}{$\begin{array}{c}\text { Cases } \\
\text { (all<1 year old) } \\
(n=469)\end{array}$} & \multicolumn{2}{|c|}{$\begin{array}{c}\text { Cases } \\
0-3 \text { months old } \\
(n=354)\end{array}$} & \multicolumn{2}{|c|}{$\begin{array}{c}\text { Cases } \\
4^{-11 \text { months old }} \\
(n=115)\end{array}$} & \multirow[t]{2}{*}{$\mathrm{p}$ value } \\
\hline & & Number & $\%$ & Number & $\%$ & Number & $\%$ & \\
\hline \multicolumn{9}{|l|}{ Demographic } \\
\hline \multirow{2}{*}{ Sex $(n=469)$} & Female & 222 & 47.3 & 165 & 46.6 & 57 & 49.6 & \multirow{2}{*}{0.593} \\
\hline & Male & 247 & 52.7 & 189 & 53.4 & 58 & 50.4 & \\
\hline \multicolumn{9}{|l|}{ Laboratory components } \\
\hline \multirow{3}{*}{$\begin{array}{l}\text { Nasopharyngeal specimen collection } \\
(\mathrm{n}=448)\end{array}$} & Aspirate only & 346 & 77.2 & 252 & 75.4 & 94 & 82.5 & \multirow{3}{*}{0.321} \\
\hline & Swab only & 80 & 17.9 & 64 & 19.2 & 16 & 14.0 & \\
\hline & Both & 22 & 4.9 & 18 & 5.4 & 4 & 3.5 & \\
\hline \multirow{2}{*}{ PCR $(n=465)$} & Positive & 465 & 100.0 & 351 & 100.0 & 114 & 100.0 & \multirow{2}{*}{ NA } \\
\hline & Negative & 0 & 0.0 & 0 & 0.0 & 0 & 0.0 & \\
\hline \multirow{2}{*}{ Culture $(n=211)^{a}$} & Positive & 108 & 51.2 & 89 & 51.7 & 19 & 48.7 & \multirow{2}{*}{0.859} \\
\hline & Negative & 103 & 48.8 & 83 & 48.3 & 20 & 51.3 & \\
\hline \multicolumn{9}{|l|}{ Clinical presentation } \\
\hline \multirow{2}{*}{ Cough $(n=469)$} & Yes & 456 & 97.2 & 342 & 96.6 & 114 & 99.1 & \multirow{2}{*}{0.202} \\
\hline & No & 13 & 2.8 & 12 & 3.4 & 1 & 0.9 & \\
\hline \multirow{2}{*}{ Cough with paroxysms $(n=456)$} & Yes & 393 & 86.2 & 298 & 87.1 & 95 & 83.3 & \multirow{2}{*}{0.347} \\
\hline & No & 63 & 13.8 & 44 & 12.9 & 19 & 16.7 & \\
\hline \multirow{2}{*}{ Whooping cough $(n=269)$} & Yes & 122 & 45.4 & 91 & 47.2 & 31 & 40.8 & \multirow{2}{*}{0.415} \\
\hline & No & 147 & 54.6 & 102 & 52.8 & 45 & 59.2 & \\
\hline \multirow{2}{*}{ Post-tussive vomiting $(n=449)$} & Yes & 219 & 48.8 & 164 & 48.7 & 55 & 49.1 & \multirow{2}{*}{1.000} \\
\hline & No & 230 & 51.2 & 173 & 51.3 & 57 & 50.9 & \\
\hline Annoea $(n=166)$ & Yes & 235 & 50.4 & 193 & 55.0 & 42 & 36.5 & \\
\hline Apnoea $(n=466)$ & No & 231 & 49.6 & 158 & 45.0 & 73 & 63.5 & 0.001 \\
\hline Cyanocic $(n=167)$ & Yes & 239 & 51.2 & 192 & 54.5 & 47 & 40.9 & 0012 \\
\hline Cy & No & 228 & 48.8 & 160 & 45.5 & 68 & 59.1 & 0.013 \\
\hline Enideminlogical link $(n=157)$ & Yes & 167 & 36.5 & 124 & 35.8 & 43 & 38.7 & 061 \\
\hline Epıdemıological lınk ( $\mathrm{n}=457)$ & No & 290 & 63.5 & 222 & 64.2 & 68 & 61.3 & 0.651 \\
\hline Diagnocic by a clinician $(n=460)$ & Yes & 368 & 78.5 & 275 & 77.7 & 93 & 80.9 & 0516 \\
\hline Diagnosis dy a cimician (n=409) & No & 101 & 21.5 & 79 & 22.3 & 22 & 19.1 & 0.516 \\
\hline Reported source of infection & & & & & & & & \\
\hline Mother $(n-12)$ & Yes & 106 & 25.0 & 88 & 27.5 & 18 & 17.3 & \\
\hline Motner $(n=424)$ & No & 318 & 75.0 & 232 & 72.5 & 86 & 82.7 & 0.038 \\
\hline Father $(n=110)$ & Yes & 82 & 19.6 & 66 & 21.0 & 16 & 15.4 & 0255 \\
\hline ratrer (II=419) & No & 337 & 80.4 & 249 & 79.0 & 88 & 84.6 & 0.253 \\
\hline Sibling $(n=116)$ & Yes & 128 & 30.8 & 102 & 32.8 & 26 & 24.8 & \\
\hline SIDIIng $(n=416)$ & No & 288 & 69.2 & 209 & 67.2 & 79 & 75.2 & 0.143 \\
\hline Grandnarents $(n=400)$ & Yes & 41 & 10.0 & 33 & 10.8 & 8 & 7.7 & \\
\hline Gramuparemts (n= 409) & No & 368 & 90.0 & 272 & 89.2 & 96 & 92.3 & 0.451 \\
\hline Careoiver $(\mathrm{n}=2,0)$ & Yes & 0 & 0.0 & 0 & 0.0 & 0 & 0.0 & $N A$ \\
\hline Caregiver $(n=240)$ & No & 240 & 100.0 & 175 & 100.0 & 65 & 100.0 & NA \\
\hline
\end{tabular}

ECMO: Extracorporeal membrane oxygenation; ICU: intensive care unit; PERTINENT: Pertussis in Infants European Network. a Cultures were done for 255 cases, but the results were only available for 211 cases.

Number of cases presented for the different characteristics are those with information available. 
Hospitalised Bordetella pertussis cases aged $<1$ year by age group, sex, laboratory components, clinical presentation, most likely source of infection and severity criteria, PERTINENT study, Europe, 1 December 2015-31 December 2018 ( $\mathrm{n}=469$ cases)

\begin{tabular}{|c|c|c|c|c|c|c|c|c|}
\hline \multirow[t]{2}{*}{ Characteristic } & & \multicolumn{2}{|c|}{$\begin{array}{c}\text { Cases } \\
\text { (all <1 year old) } \\
(n=469)\end{array}$} & \multicolumn{2}{|c|}{$\begin{array}{c}\text { Cases } \\
0-3 \text { months old } \\
(n=354)\end{array}$} & \multicolumn{2}{|c|}{$\begin{array}{c}\text { Cases } \\
4-11 \text { months old } \\
(n=115)\end{array}$} & \multirow[t]{2}{*}{$\mathrm{p}$ value } \\
\hline & & Number & $\%$ & Number & $\%$ & Number & $\%$ & \\
\hline \multicolumn{9}{|l|}{ Severity criteria } \\
\hline \multirow{2}{*}{ Death $(n=466)$} & Yes & 5 & 1.1 & 5 & 1.4 & 0 & 0.0 & \multirow{2}{*}{0.340} \\
\hline & No & 461 & 98.9 & 346 & 98.6 & 115 & 100.0 & \\
\hline \multirow{2}{*}{ ICU $(n=330)$} & Yes & 83 & 25.2 & 80 & 31.5 & 3 & 3.9 & \multirow{2}{*}{0.000} \\
\hline & No & 247 & 74.8 & 174 & 68.5 & 73 & 96.1 & \\
\hline \multirow{2}{*}{ ECMO $(n=336)$} & Yes & 7 & 2.1 & 7 & 2.7 & 0 & 0.0 & \multirow{2}{*}{0.360} \\
\hline & No & 329 & 97.9 & 251 & 97.3 & 78 & 100.0 & \\
\hline \multirow{2}{*}{ Pneumonia $(n=327)$} & Yes & 14 & $4 \cdot 3$ & 12 & 4.8 & 2 & 2.7 & \multirow{2}{*}{0.745} \\
\hline & No & 313 & 95.7 & 240 & 95.2 & 73 & 97.3 & \\
\hline \multirow{2}{*}{ Encephalopathy $(n=327)$} & Yes & 3 & 0.9 & 3 & 1.2 & 0 & 0.0 & \multirow{2}{*}{1.000} \\
\hline & No & 324 & 99.1 & 249 & 98.8 & 75 & 100.0 & \\
\hline \multirow{2}{*}{ Seizure $(n=328)$} & Yes & 10 & 3.0 & 8 & 3.2 & 2 & 2.7 & \multirow{2}{*}{1.000} \\
\hline & No & 318 & 97.0 & 245 & 96.8 & 73 & $97 \cdot 3$ & \\
\hline \multirow{2}{*}{ Eating difficulties $(n=269)$} & Yes & 62 & 23.0 & 50 & 25.4 & 12 & 16.7 & \multirow{2}{*}{0.144} \\
\hline & No & 207 & 77.0 & 147 & 74.6 & 60 & 83.3 & \\
\hline \multirow{2}{*}{ Kidney failure $(n=267)$} & Yes & 4 & 1.5 & 4 & 2.0 & 0 & 0.0 & \multirow{2}{*}{0.576} \\
\hline & No & 263 & 98.5 & 192 & 98.0 & 71 & 100.0 & \\
\hline \multirow{2}{*}{ Dehydration $(n=300)$} & Yes & 12 & 4.0 & 11 & 4.8 & 1 & 1.4 & \multirow{2}{*}{0.305} \\
\hline & No & 288 & 96.0 & 216 & 95.2 & 72 & 98.6 & \\
\hline
\end{tabular}

ECMO: Extracorporeal membrane oxygenation; ICU: intensive care unit; PERTINENT: Pertussis in Infants European Network. Number of cases presented for the different characteristics are those with information available.

to 2017. Pertussis cases are described by severity and other characteristics. VE results are not presented as VE will be assessed once the required sample size is reached.

\section{Methods}

\section{Study design and setting}

The PERTINENT coordination selected seven study sites (Czech Republic; France; Ireland; Italy; Norway; Catalonia, Spain and Navarra, Spain) willing to participate and able to comply with the generic PERTINENT sentinel surveillance protocol [16] and laboratory guidelines [17] and to estimate the participating hospitals' catchment population. We organised site visits and a laboratory workshop to ensure the harmonisation of site-specific protocols allowing pooling of sites' data.

From December 2015 to September 2016, study sites progressively implemented active surveillance in 41 hospitals. A large proportion of hospitals were situated in France ( $n=21$ hospitals) and other countries had one to six participating hospitals. Each site complied with the local ethical procedures. In May 2018, the number of participating hospitals was reduced to
37 after withdrawal of four Norwegian hospitals (Table 1). All sites use the acellular pertussis vaccine for the primary series in infants, but national vaccine recommendations and primary schedules vary across sites (Table 1 and supplementary Table S4).

\section{Case identification and recruitment}

The study population consisted of all infants aged less than 1 year, likely to be hospitalised in one of the participating hospitals if developing pertussis-like symptoms.

To maximise the sensitivity of the surveillance, we raised hospital physicians' awareness of pertussis clinical presentation [3] and asked them to test all infants presenting at hospital with pertussis-like symptoms. 'Typical' pertussis presentation was defined either by a presence of apnoea; or by a cough associated with at least one of paroxysms, whoop or post-tussive vomiting. When physicians suspected pertussis even though some typical symptoms were missing, pertussis was considered as 'atypical'.

We identified all infants attending the hospital who were tested for pertussis and invited their parents to participate in the study. When required by the local 


\section{FIGURE 2}

Hospitalised Bordetella pertussis cases aged $<1$ year by month of symptom onset, PERTINENT study, Europe, 1 December 2015-31 December $2018\left(n=464\right.$ cases $\left.^{\mathrm{a}}\right)$

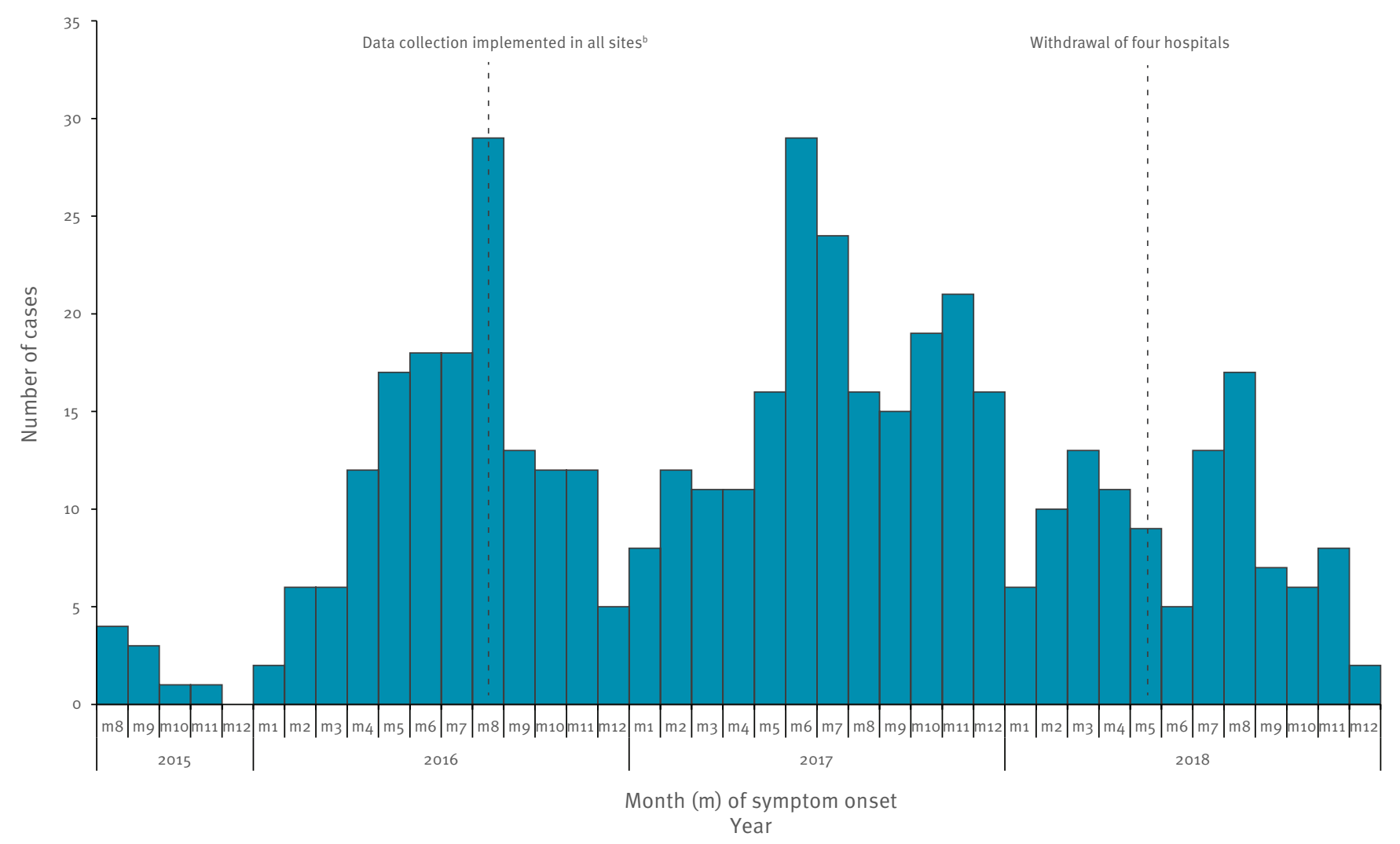

PERTINENT: Pertussis in Infants European Network.

a Five patients with missing date of symptom onset are not included in the figure.

${ }^{b}$ Data collection started in 2015, but was fully operational in all sites in August 2016 only.

ethical committee, parents or legal guardians were requested to provide an informed consent.

We excluded all patients with missing or pending laboratory results, testing positive to other Bordetella species than $B$. pertussis or whose legal guardian was unwilling to participate or unable to communicate and give consent. All laboratory-confirmed B. pertussis cases aged less than 1 year at the time of hospitalisation were included in the study (Figure 1).

\section{Definitions}

We defined a laboratory-confirmed $B$. pertussis case as an infant attending one of the participating hospitals (irrespective of the length of stay), aged less than 1 year and testing positive for $B$. pertussis by PCR (DNA detection of $B$. pertussis using PCR or real-time PCR in a nasopharyngeal aspirate or swab) or culture (isolation of $B$. pertussis from the prior-mentioned clinical specimen) regardless of the clinical criteria. We defined a severe case as a case admitted to the intensive care unit (ICU).
We defined a likely source of infection as a person with a cough who had contact with the case in the 7 to 20 days before the date of symptom onset of the case. For each pertussis vaccine dose, we defined an infant as vaccinated if she/he had received the dose of interest $>14$ days before symptom onset. Unvaccinated infants were those who had not received any dose or who had received the first dose $\leq 14$ days before symptom onset.

\section{Laboratory methods}

To ensure an accurate identification of the Bordetella species, the PERTINENT laboratory guidelines recommend a diagnostic algorithm for DNA detection of Bordetella including a series of three PCRs: a triplex real-time PCR targeting IS481 gene (in $B$. pertussis, holmesii and some bronchiseptica strains), pIS1001 (B. parapertussis) and RNase P as the human internal control; followed by two confirmatory singleplex tests targeting $p t x A-\operatorname{Pr}(B$. pertussis) and hIS1001 (B. holmesii) genes [17]. 


\section{FIGURE 3}

Hospitalised Bordetella pertussis cases aged $<1$ year by age in months, PERTINENT study, Europe, 1 December 2015-31 December 2018 ( $n=469$ cases)

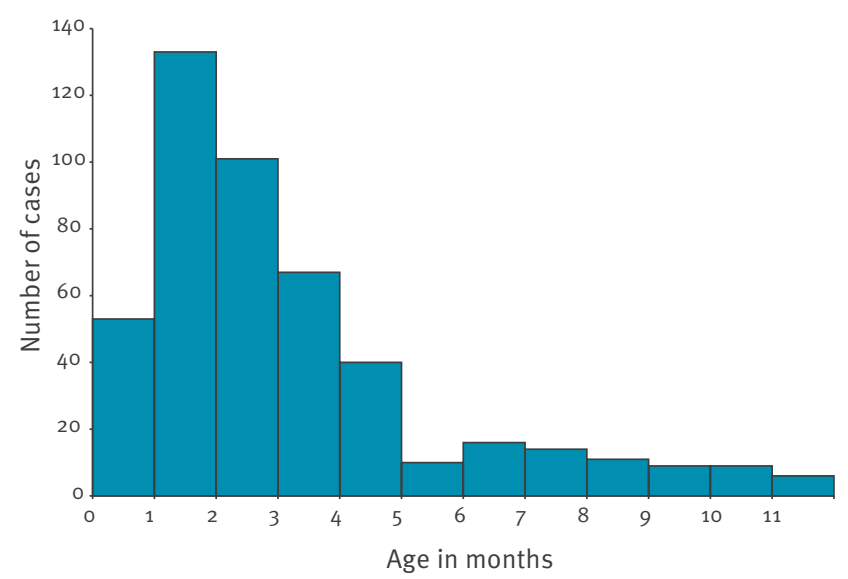

PERTINENT: Pertussis in Infants European Network.

\section{Data collection}

Using a standard questionnaire, all sites collected a common set of information: demographic, epidemiological, clinical, laboratory data, vaccination status of the infant and household members, risk and protective factors and suspected source of infection. The list of potential sources of infection included close relatives and caregivers. Each study site translated the questionnaire available in English in its country's language. Data were collected through review of clinical case notes, extraction from patient registries or, if not available, interviews with parents or legal guardians.

\section{Denominators}

The denominator was the estimated population of infants aged less than 1 year likely to receive care at hospitals participating in the study. The methods to estimate the hospitals' catchment population varied across sites (Table 1). Throughout the study period, we adjusted the catchment population to the loss of the four Norwegian hospitals.

\section{Analysis}

We described cases by age, clinical presentations, risk and protective factors, severity. We used median for continuous variables (age in months, gestational age and weight at birth) and frequencies for categorical variables. We estimated the incidence by site and year. In four sites, the protocol was implemented during 2016 and we estimated 2016 incidence prorata temporis. We calculated incidence rate ratios (IRR) for 2018 compared with 2017 .

We used Fisher's exact test to compare clinical signs and symptoms by age group and characteristics of cases admitted to ICU with those not admitted to ICU.

\section{Ethical statement}

The planning, conduct and reporting of the study was in line with the Declaration of Helsinki [18]. Ethical approval was not needed in Navarra as the PERTINENT study came under the umbrella of the mandatory surveillance system. Other study sites sought ethical approval from a review board according to country-specific regulations (Catalonia:PIC-31-16, Czech Republic:SZU/05992/2019, France: $\mathrm{n}^{\circ} 449199 \mathrm{~V} 1$, Ireland: REC reference number 16.058 and Gen/499/16, Italy:Bambino Gesù Children's Hospital Ethical Committee: protocol n. 1064_OPBG_2016, Norway: REC register number 2015/956).

\section{Results}

\section{Description of cases}

Of the 2,114 infants tested for $B$. pertussis, 2,081 had laboratory results available (98\%) (Figure 1). We excluded 39 cases caused by other Bordetella species $(2 \%)$. A total of 469 infants were positive for $B$. pertussis $(23 \%)$ including one co-infection with $B$. pertussis and parapertussis. The number of pertussis cases by site ranged from seven in Norway to 199 cases in France (Table 1).

Among the 469 laboratory-confirmed $B$. pertussis cases, information on the sample type was available for 448 , with $368(82 \%)$ having had a nasopharyngeal aspirate, 102 (23\%) a nasopharyngeal swab and 22 (5\%) both. Of all 469 confirmed cases, 465 had a PCR (99\%) and 255 a culture done (54\%). All PCRs and 108 of 211 culture results available $(51 \%)$ were positive to $B$. pertussis (Table 2 ).

Of the 469 cases, 247 were males (53\%). The median age was 2.5 months (range: $0-11.6$; interquartile range $(I Q R): 2.5)$. The median weight at birth was $3,280 \mathrm{~g}$ (range:700-4,925; IQR:720). The median gestational duration was 39 weeks (range: 25-42; IQR: 2).

The number of reported cases by month of symptom onset (Figure 2) was highest in August $2016(n=29)$ and in June $2017(n=29)$. Excluding the first months of progressive surveillance implementation, less cases were observed at the transition between years, such as December $2016(n=5)$, January $2018(n=6)$ and December $2018(n=2)$, but not always, as for example June $2018(n=5)$.

The highest number of cases was reported in the age group $0-2$ months $(n=287)$, with a peak in the second month of life $(n=133)$, then the number decreased by age in months (Figure 3).

Of the 469 cases, 456 (97\%) had a cough, among whom $86 \%$ had paroxysm. For those who had cough and additional information on post-tussive vomiting $(n=449)$ and whoop $(n=269), 49 \%$ had post-tussive vomiting and $45 \%$ whoop. For cases with available data on apnoea $(n=466), 235(50 \%)$ had apnoeic episodes 
(Table 2), including six cases without cough. Pertussis was typical in 439 cases (94\%) (Figure 1). Thirty cases had an atypical pertussis clinical presentation (6\%), among whom 20 had a cough only, three had cough and cyanosis only, two had a cyanosis only and five had documented absence of all pertussis-like symptoms. Four of these atypical pertussis cases had at least one missing clinical information.

Among 354 cases aged 0-3 months, $250(71 \%)$ had either apnoea or cyanosis compared with 62 (54\%) among 115 cases aged $4-11$ months $(p=0.001)$ (Table 2).

Clinical presentation did not differ between vaccinated and not vaccinated infants (data not shown). Of all cases, $290(62 \%)$ were eligible for vaccination. Of the 273 with information on vaccination status, 106 (39\%) were vaccinated: 59 had received one dose, 39 two doses and eight three doses of pertussis vaccine. Of the 30 atypical cases, 18 were eligible for vaccination and, of the 17 with related data, about half $(n=9)$ had received one or two doses.

\section{Potential source of infection}

Information on the potential source of infection was available for 441 cases ( $94 \%$ ). Infants' sibling was mentioned for 128 cases (31\%), the mother for 106 cases (25\%), the father for 82 cases $(20 \%)$ and the grandparents for 41 cases (10\%) (Table 2).

\section{Severity}

Five $(1 \%)$ of 466 cases with information died. They were aged from 2 weeks to 10 weeks and not vaccinated against pertussis. In two of the five cases, the mother was vaccinated 5 to 10 years earlier. For one case, the mother was vaccinated 5 days before delivery. Vaccination status of the mother was unknown for the remaining two cases.

Eighty-three cases (25\%) were admitted to ICU, including seven cases treated with extracorporeal membrane oxygenation (ECMO) and the five infants who died (Table 2). Among infants aged o-3 months, 80 (32\%) were admitted to ICU compared with three (4\%) among infants aged $4^{-11}$ months ( $<$ <0.001) (Table 2). The median age in months was 1.4 (range: $0-4.6 ; \mathrm{IQR}: 1.3$ ) in cases who required ICU admission and 2.8 (range: $0.1-$ 11.6; IQR: 2.7 ) in those who did not ( $p<0.001)$. Of ICU cases, $66(80 \%)$ had either apnoea or cyanosis compared with $160(65 \%)$ in non-ICU cases $(p=0.014)$. The proportion of cases with comorbidities was similar between the two groups. Among cases with available gestational week, 21 of the $82 \mathrm{ICU}$ cases (26\%) and 26 of 247 non-ICU cases (11\%) were preterm infants born before gestational week $37(p=0.002)$ (Table 3$)$.

Among infants eligible for vaccination, seven of the 25 ICU cases (28\%) and 65 of the 163 non-ICU cases (40\%) were vaccinated with at least one dose of pertussis vaccine $(p=0.279)$. None of the ICU cases and 35 of the
non-ICU cases (21\%) had received two or more doses of pertussis vaccine $(p=0.005)$.

\section{Incidence by site}

Incidence rate ratios between 2018 and 2017 were 1.43 in Czech Republic $(p=0.468), 0.25$ in Catalonia $(p=0.002), 0.71$ in France $(p=0.0335), 0.14$ in Ireland $(p=0.002), 0.63$ in Italy $(p=0.053), 0.21$ in Navarra $(p=0.148)$ and zero in Norway (Table 1$)$.

\section{Other Bordetella species}

Five of the seven sites provided information on other Bordetella species. We identified 40 respiratory infections caused by other Bordetella species, including 17 B. parapertussis (cases aged 6 to 51 weeks) and four $B$. holmesii (cases aged 7 to 14 weeks). Of the $17 B$. parapertussis cases, two did not have typical pertussislike symptoms but only cough and three of 12 B. parapertussis cases with information were admitted to ICU. Patients infected with $B$. holmesii had pertussis-like symptoms and did not require ICU admission. Among infants eligible for vaccination, eight of $13 B$. parapertussis cases and two of three $B$. holmesii cases were vaccinated with at least one dose of pertussis vaccine.

\section{Discussion}

Over the three pilot years of the PERTINENT active hospital-based surveillance system, we identified 469 laboratory-confirmed $B$. pertussis cases. We observed a higher incidence in 2017 and a decrease in 2018 in all but one study site. In 2016 and 2017, most of the cases were reported during summer. One of four pertussis laboratory-confirmed hospitalised cases was admitted to ICU. The highest proportion of ICU cases was in infants aged $0-3$ months. Five infants died, all were unvaccinated: either too young to be vaccinated or in the month of life targeted for the first dose of the primary schedule.

Despite standard protocols proposed, the PERTINENT pilot surveillance still has limits to consider when examining the findings. Different methods were used by study sites for estimating hospital catchment population. This may have biased the measured incidence and made annual comparison between sites difficult. However, those methods did not change over time and allowed to compute and compare incidence by sites. During the pilot phase, one country had to decrease the number of participating hospitals and we adjusted the incidence denominator accordingly.

Among the five sites that provided information on other Bordetella species, the proportion of patients with other Bordetella species among those testing positive for any Bordetella species was 11\% (40/357) on average, ranging from o of 25 to 5 of 18 . Two sites were not able to differentiate $B$. pertussis from the other Bordetella species that may induce a similar respiratory presentation upon infection. This likely decreased the specificity of the laboratory testing and increased reported incidences. 
TABLE 3

Hospitalised Bordetella pertussis cases aged < 1 year by ICU admission, clinical presentation, comorbidities and risk/ protective factors, PERTINENT study, Europe, 1 December 2015-31 December 2018 (n=330 cases ${ }^{\mathrm{a}}$ )

\begin{tabular}{|c|c|c|c|c|c|c|}
\hline \multirow[t]{2}{*}{ Characteristic } & & \multicolumn{2}{|c|}{$\begin{array}{l}\text { Admitted to the ICU } \\
\qquad(\mathrm{n}=83)\end{array}$} & \multicolumn{2}{|c|}{$\begin{array}{l}\text { Not admitted to the ICU } \\
\qquad(n=247)\end{array}$} & \multirow[t]{2}{*}{$\mathrm{p}$ value } \\
\hline & & Number & $\%$ & Number & $\%$ & \\
\hline \multicolumn{7}{|l|}{ Clinical presentation } \\
\hline \multirow{2}{*}{ Cough $(n=330)$} & Yes & 83 & 100.0 & 238 & 96.4 & \multirow{2}{*}{0.119} \\
\hline & No & o & 0.0 & 9 & 3.6 & \\
\hline \multirow{2}{*}{ Paroxysms among those with cough $(n=321)$} & Yes & 73 & 88.0 & 203 & 85.3 & \multirow{2}{*}{0.714} \\
\hline & No & 10 & 12.0 & 35 & 14.7 & \\
\hline \multirow{2}{*}{ Whoop $(n=252)$} & Yes & 17 & 43.6 & 101 & 47.4 & \multirow{2}{*}{0.728} \\
\hline & No & 22 & 56.4 & 112 & 52.6 & \\
\hline \multirow{2}{*}{ Post-tussive vomiting $(n=320)$} & Yes & 45 & 54.2 & 110 & 46.4 & \multirow{2}{*}{0.251} \\
\hline & No & 38 & 45.8 & 127 & 53.6 & \\
\hline \multirow{2}{*}{ Apnoea $(n=328)$} & Yes & 55 & 66.3 & 128 & 52.2 & \multirow{2}{*}{0.030} \\
\hline & No & 28 & 33.7 & 117 & 47.8 & \\
\hline \multirow{2}{*}{ Cyanosis $(n=329)$} & Yes & 59 & 71.1 & 106 & 43.1 & \multirow{2}{*}{0.000} \\
\hline & No & 24 & 28.9 & 140 & 56.9 & \\
\hline \multicolumn{7}{|l|}{ Comorbidities } \\
\hline \multirow{2}{*}{ Cardiovascular $(n=330)$} & Yes & 4 & 4.8 & 6 & 2.4 & \multirow{2}{*}{0.278} \\
\hline & No & 79 & 95.2 & 241 & 97.6 & \\
\hline \multirow{2}{*}{ Respiratory $(n=330)$} & Yes & 2 & 2.4 & 4 & 1.6 & \multirow{2}{*}{0.644} \\
\hline & No & 81 & 97.6 & 243 & 98.4 & \\
\hline \multirow{2}{*}{ Immunodeficiencies $(n=129)$} & Yes & 0 & 0.0 & 0 & 0.0 & \multirow{2}{*}{ NA } \\
\hline & No & 57 & 100.0 & 72 & 100.0 & \\
\hline \multicolumn{7}{|l|}{ Risk/protective factors } \\
\hline \multirow{2}{*}{ Breastfeeding $(n=327)$} & Yes & 50 & 62.5 & 166 & 67.2 & \multirow{2}{*}{0.497} \\
\hline & No & 30 & 37.5 & 81 & 32.8 & \\
\hline \multirow{2}{*}{ Premature 37 weeks $(n=329)$} & Yes & 21 & 25.6 & 26 & 10.5 & \multirow{2}{*}{0.002} \\
\hline & No & 61 & 74.4 & 221 & 89.5 & \\
\hline
\end{tabular}

ICU: intensive care unit; PERTINENT: Pertussis in Infants European Network.

330 cases of 469 had information on ICU or non-ICU admission.

Numbers of cases with available information are presented.

PERTINENT laboratories did not so far sequence $B$. pertussis isolates, which prevented detection of changes in the pathogen. Isolates were stored in optimal conditions to be analysed later on.

Severity of the disease remains difficult to interpret because of different severity ascertainment and clinical practices by country and by hospital. Hospitalised cases are usually severe pertussis cases, however, the probability of being hospitalised for pertussis-like symptoms is heterogeneous across sites due to different referral to hospital practices. In France, any infant aged less than 3 months with pertussis-like symptoms will be admitted regardless of the severity of the disease. In contrast, a large proportion of Norwegian infants will first attend the 'out-of-office emergency primary care' services (legevakt) and only severe cases will be transferred to hospitals. This likely affected the comparison of clinical signs and incidence rate of hospitalised severe cases between sites.
The likely source of infection was ascertained by family interviews, which may require caution in interpreting the results. Caregivers were not reported as probable sources for any of the reported pertussis cases. As parents self-reported the likely source of infection, they may have better remembered signs of coughing in the household members than among the infant's caregivers. Additional questions, laboratory confirmation in suspected sources of infection, different study designs with increased data completeness are needed to identify source of infection.

Despite using a standard protocol in all sites, data completeness still needs improvement. In severity variables, completeness ranged from $57 \%$ (267/469) to $72 \%$ (336/469), except for death ascertainment with a completeness $>99 \%$ (Table 2 ).

Taking the above limitations into account, our results suggest a decrease in 2018 in pertussis incidence 
compared with 2017, across all sites except for the Czech Republic site where incidence remained stable. The low number of study sites does not allow to extrapolate results to national nor EU/EEA level. However, incidences reported to the European Surveillance System (TESSy) by five of the six countries involved in PERTINENT also suggest a decrease. Incidences reported for hospitalised and non-hospitalised cases in 2018 ranged from 42.9 to 85.3 per 100,000 infants in all infants aged less than 1 year, as compared with a range of 12.9 to 87.6 per 100,000 infants in PERTINENT for hospitalised infants only (excluding Norway where zero cases were observed) suggesting a better sensitivity of the PERTINENT data.

We suggest that the summer peak observed in reported cases might possibly reflect the seasonality of the disease. It is unlikely that this was due to improved diagnosis in those months as the PERTINENT surveillance system was stable over time. An increase in pertussis cases during summer was previously reported. In the Netherlands where pertussis is a statutory notifiable disease, the annual peak incidence of notifications for all age groups (0-4; 5-12; 13-18 and 19-99years) between 1996 and 2006 was in August [19]. More recently, using the notifiable infectious disease reporting system in China from January 2004 to May 2018, Wang suggested a seasonality in pertussis cases and a summer peak with a maximum in August [20].

Thirty cases did not have a typical pertussis clinical presentation including four with at least one clinical sign not documented and five cases with documented absence of all pertussis-like symptoms (reason for hospitalisation unknown). The EU case definition for pertussis was revised in June 2018 [21] to draw attention on atypical symptoms in adults, adolescents or vaccinated children. Our results may suggest the existence of atypical pertussis in infants [3] and highlight the need to raise clinicians' awareness about possible under-diagnosis of pertussis in that age group. The World Health Organization and United States Centers for Disease Control and Prevention pertussis case definitions do not include isolated apnoea or cyanosis in the clinical criteria for pertussis surveillance. In our study, pertussis cases aged less than 3 months were more likely to present with apnoea or cyanosis, which supports the inclusion of these clinical criteria in the pertussis case definition.

Our results suggest that cases admitted to ICU were younger and less vaccinated than non-ICU cases. In the PERTINENT hospital-based sentinel network, four pertussis deaths were reported in 2017 across the seven study sites. As pertussis cases identified in the study were followed up during hospitalisation, we expect no under-reporting of deaths. Among pertussis cases aged less than 1 year reported to TESSy, there were three pertussis deaths in 2017 across the 29 EU/EEA reporting countries [2]. This may suggest that detection of pertussis hospital death is more sensitive in the PERTINENT system. As described in other systems, deaths may be under-ascertained in routine hospital based surveillance in EU/EEA countries [22,23].

The most likely source of infection reported by the parents was firstly the patient's siblings followed by the mother. Recent studies have also shown an increased risk of transmission to siblings of primary cases [24] and a shift in the source of infection from the mother to the siblings [25]. This may be a consequence of vaccinating the mother either before, during or after pregnancy (cocooning strategy), therefore preventing transmission to infants.

Bordetella species can be isolated from both nasopharyngeal swabs or aspirates but a $15 \%$ gain in the isolation rate can be obtained by using aspirates in neonates and infants [26]. In our study, we reached a good quality of specimen collection with a high proportion of nasopharyngeal aspirates (82\%). We identified 40 infections caused by other Bordetella species, including four with $B$. holmesii, which is rarely isolated in infants [27]. Even though other Bordetella species are not directly targeted by pertussis-containing vaccine, the later may also induce some cross-immunity for specific Bordetella species [28].

\section{Conclusions}

This pilot project shows that enhanced pertussis surveillance in Europe is possible. The generic protocol presented some challenges and efforts by all partners were needed to improve data quality and laboratory procedures but we believe this allowed to pool sites' data to better describe hospitalised laboratoryconfirmed pertussis cases, as these were recruited using the same criteria across six EU/EEA countries. However, a larger sustained project is needed with additional countries to ensure representativeness in Europe and a particular emphasis on harmonisation of laboratory methods. In the future, this surveillance network should allow monitoring emergence of atypical pertussis presentation, identifying upcoming pertussis epidemic cycles and comparing incidence over time in Europe according to immunisation strategies. It will also allow measuring the effectiveness of infants' and mothers' vaccination.

\section{PERTINENT group}

Epiconcept, France: Lore Merdrignac, Epiconcept; Camelia Savulescu, Epiconcept; Marta Valenciano, Epiconcept; Alain Moren, Epiconcept.

Czech Republic: Pavla Křǐžová, National Institute of Public Health, Prague; Kateřina Fabiánová, National Institute of Public Health, Prague; Jana Zavadilová, National Institute of Public Health, Prague; Zuzana Blechová, University Hospital $\mathrm{Na}$ Bulovce, Prague; Květa Bláhová, University Hospital Motol, Prague; Pavel Kosina, University Hospital, Hradec Králové; Josef Sýkora, University Hospital, Pilsen; Alena Holčíková, University Hospital, Brno; Petr Širůček, University Hospital, Ostrava. 
France: Daniel Lévy-Brühl, Santé Publique France, SaintMaurice; Fatima Aït El Belghiti, Santé Publique France, Saint-Maurice; Adèle Grembombo, Santé Publique France, Saint-Maurice; Sophie Guillot, Institut Pasteur, Paris; Sylvain Brisse, Institut Pasteur, Paris; Julie Toubiana, Institut Pasteur, Paris.

Ireland: Suzanne Cotter, HSE-Health Protection Surveillance Centre, Dublin; Jane Murphy, Temple Street Children's University Hospital, Dublin; Robert Cunney, Temple Street Children's University Hospital, Dublin; Norma O'Shaughnessy, Temple Street Children's University Hospital, Dublin; Adele Habington, Our Lady's Children's hospital Crumlin, Dublin; Niamh O'Sullivan, Our Lady's Children's hospital Crumlin, Dublin.

Italy: Elisabetta Pandolfi, Bambino Gesù Children Hospital, Rome; Alberto E Tozzi, Bambino Gesù Children Hospital, Rome; Caterina Rizzo, Bambino Gesù Children Hospital, Rome; Luisa Russo, Bambino Gesù Children Hospital, Rome; Ilaria Campagna, Bambino Gesù Children Hospital, Rome; Francesco Gesualdo, Bambino Gesù Children Hospital, Rome; Sara Ciampini, Bambino Gesù Children Hospital, Rome; Valentina Annarosa Ferro, Bambino Gesù Children Hospital, Rome; Elena Boccuzzi, Bambino Gesù Children Hospital, Rome.

Norway: Elmira Flem, Norwegian Institute of Public Health, Oslo; Håkon Bøås, Norwegian Institute of Public Health, Oslo; Terese Bekkevold, Norwegian Institute of Public Health, Oslo; Liliana Vazquez Fernandez, Norwegian Institute of Public Health, Oslo.

Catalonia, Spain: Carmen Muñoz-Almagro, Instituto de Recerca Pediatrica Hospital Sant Joan de Deu, Barcelona; Universitat Internacional de Catalunya and CIBER of Epidemiology and Public Health CIBERESP; Cristina Esteva, Instituto de Recerca Pediatrica Hospital Sant Joan de Deu, Barcelona; CIBER of Epidemiology and Public Health CIBERESP; Miguel Lanaspa Perez, Instituto de Recerca Pediatrica Hospital Sant Joan de Deu, Barcelona; Mireia Jané, Public Health Agency of Catalonia, Barcelona; CIBER of Epidemiology and Public Health CIBERESP; University of Barcelona; Gloria Carmona, Public Health Agency of Catalonia, Barcelona; Lesly Acosta, Universitat Politècnica de Catalunya - BarcelonaTech (UPC), Public Health Agency of Catalonia, Barcelona; Yolanda Jordan Garcia, Instituto de Recerca Pediatrica Hospital Sant Joan de Deu, Barcelona; CIBER of Epidemiology and Public Health CIBERESP.

Navarra, Spain: Manuel García Cenoz, Instituto de Salud Pública de Navarra, IdiSNA - Navarre Institute for Health Research, Pamplona; Ana Navascués, Complejo Hospitalario de Navarra, Pamplona; Leticia Fernandino Zubieta, Instituto de Salud Pública de Navarra, IdiSNA - Navarre Institute for Health Research, Pamplona; Jesús Castilla, Instituto de Salud Pública de Navarra, IdiSNA - Navarre Institute for Health Research, Pamplona.

Sentinelles, France: Thomas Hanslik, Sorbonne Université, INSERM, Institut Pierre Louis d'Epidémiologie et de Santé Publique (IPLESP UMRS 1136), Paris.

ECDC: Sabrina Bacci, European Centre for Disease Prevention and Control, Stockholm, Sweden; Gianfranco Spiteri, European Centre for Disease Prevention and Control, Stockholm, Sweden.

\section{Acknowledgements}

We are grateful to all patients and their legal tutor, nurses, clinicians, microbiologists and epidemiologists from the seven study sites who actively participated in the study. Thanks to the epidemiologists, clinicians, microbiologists, biostatisticians and other researchers from the four expert sites who were engaged in the study: Wiebke Hellenbrand, Kai Michaelis, Robert Koch Institute, Berlin, Germany; Odette Popovici, National Institute of Public Health, Bucharest, Romania; Bernice Aronsson, Public Health Agency of Sweden, Stockholm, Sweden; Heather Murdoch, Alison Smith-Palmer, Joe Jasperse, Health Protection Scotland, Glasgow, Scotland.

Funding: The European Centre for Disease Prevention and Control (ECDC) funded the PERTINENT study (Framework contract $\left.n^{\circ} E C D C / 2015 / 017\right)$.

\section{Conflict of interest}

None declared.

\section{Authors' contributions}

Lore Merdrignac: coordination of PERTINENT network, study design, analysis of data, interpretation of results, manuscript writing.

Marta Valenciano, Alain Moren: study design, interpretation of results, contribution to manuscript writing.

Thomas Hanslik: interpretation of results, contribution to manuscript writing.

Authors affiliated to study sites: Fatima Aït El Belghiti, Elisabetta Pandolfi, Mireia Jané, Jane Murphy, Kateřina Fabiánová, Manuel García Cenoz, Elmira Flem, Sophie Guillot, Alberto E Tozzi, Gloria Carmona, Adele Habington, Jana Zavadilová, Ana Navasués, Håkon Bøås, Daniel LévyBrühl, Beatrice Ferretti, Miguel Lanaspa, Niam O'Sullivan, Pavla Kř́̌žová, Leticia Fernandino, Terese Bekkevold, Carmen Muñoz-Almagro and PERTINENT group: data collection, data validation, results interpretation, review of manuscript.

ECDC: Sabrina Bacci, Gianfranco Spiteri: study design, interpretation of results, review of manuscript.

\section{References}

1. Yeung KHT, Duclos P, Nelson EAS, Hutubessy RCW. An update of the global burden of pertussis in children younger than 5 years: a modelling study. Lancet Infect Dis. 2017;17(9):9748o. https://doi.org/10.1016/S1473-3099(17)30390-0 PMID: 28623146

2. European Centre for Disease Prevention and Control (ECDC).Pertussis - Annual Epidemiological Report for 2017. Stockholm: ECDC; 2019. Available from: https://www.ecdc.europa.eu/en/publications-data/ pertussis-annual-epidemiological-report-2017

3. Mattoo S, Cherry JD. Molecular pathogenesis, epidemiology, and clinical manifestations of respiratory infections due to Bordetella pertussis and other Bordetella subspecies. Clin Microbiol Rev. 2005;18(2):326-82. https://doi.org/10.1128/ CMR.18.2.326-382.2005 PMID: 15831828

4. Vittucci AC, Spuri Vennarucci V, Grandin A, Russo C, Lancella L, Tozzi AE, et al. Pertussis in infants: an underestimated disease. BMC Infect Dis. 2016;16(1):414. https://doi.org/10.1186/ S12879-016-1710-0 PMID: 27528377

5. European Centre for Disease Prevention and Control (ECDC). Pertussis - Annual Epidemiological Report 2016. Stockholm: ECDC; 2016. Available from: https://www.ecdc.europa.eu/en/publications-data/ pertussis-annual-epidemiological-report-2016-2014-data

6. World Health Organization (WHO). WHO-UNICEF estimates of DTP 3 coverage. Geneva: WHO. [Accessed 21 Nov 2019]. Available from: https://apps.who.int/immunization monitoring/globalsummary/timeseries/tswucoveragedtp3.html

7. Tan T, Dalby T, Forsyth K, Halperin SA, Heininger U, Hozbor D, et al. Pertussis Across the Globe: Recent Epidemiologic Trends From 2000 to 2013. Pediatr Infect Dis J. 2015;34(9):e222-32. 
https://doi.org/10.1097/INF.0000000000000795 PMID: 26376316

8. Heininger U, André P, Chlibek R, Kristufkova Z, Kutsar K, Mangarov A, et al. Comparative Epidemiologic Characteristics of Pertussis in 10 Central and Eastern European Countries, 2000-2013. PLoS One. 2016;11(6):e0155949. https://doi. org/10.1371/journal.pone.0155949 PMID: 27257822

9. European Centre for Disease Prevention and Control (ECDC). Surveillance systems overview for 2016. Stockholm: ECDC 2018. Available from: https://www.ecdc.europa.eu/en/ publications-data/surveillance-systems-overview-2016

10. European Commission. Commission Implementing Decision of 8 August 2012. Official Journal of the European Union. Luxembourg: Publications Office of the European Union. 27.9.2012: L 262/1. Available from: https://eur-lex.europa.eu/ LexUriServ/LexUriServ.do?uri=0J:L:2012:262:0001:0057:EN:P DF

11. European Centre for Disease Prevention and Control (ECDC). Expert consultation on pertussis - Barcelona, 20 November 2012. Stockholm: ECDC; 2014. Available from: https://www. ecdc.europa.eu/sites/default/files/media/en/publications/ Publications/pertussis-meeting-2012.pdf

12. Abu-Raya B, Bettinger JA, Vanderkooi OG, Vaudry W, Halperin SA, Sadarangani M,, et al. Burden of Children Hospitalized With Pertussis in Canada in the Acellular Pertussis Vaccine Era, 1999-2015. J Pediatric Infect Dis Soc. 2020;9(2):118-27. https://doi.org/10.1093/jpids/piy128 PMID: 30535079

13. Tubiana S, Belchior E, Guillot S, Guiso N, Lévy-Bruhl D, Renacoq Participants. Monitoring the Impact of Vaccination on Pertussis in Infants Using an Active Hospital-based Pediatric Surveillance Network: Results from 17 Years' Experience, 19962012, France. Pediatr Infect Dis J. 2015;34(8):814-20. https:// doi.org/10.1097/INF.0000000000000739 PMID: 25955837

14. McRae JE, Quinn HE, Saravanos GL, McMinn A, Britton PN, Wood N, et al. Paediatric Active Enhanced Disease Surveillance (PAEDS) annual report 2016: Prospective hospital-based surveillance for serious paediatric conditions. Commun Dis Intell (2018). 2019;43. https://doi.org/10.33321/cdi.2019.43.5 PMID: 30727704

15. Skoff TH, Baumbach J, Cieslak PR. Tracking Pertussis and Evaluating Control Measures through Enhanced Pertussis Surveillance, Emerging Infections Program, United States. Emerg Infect Dis. 2015;21(9):1568-73. https://doi.org/10.3201/ eid2109.150023 PMID: 26291475

16. PERTINENT Network. Pertussis enhanced surveillance: Generic Protocol v 1.5. Paris: Epiconcept; 2018. Available from: https:// docs.google.com/a/epiconcept.fr/viewer?a=v\&pid=sites\&srcid $=Z_{X B p Y}$ guY2VwdC 5 mcnxwZXJoaW5lbnR8Z3g6MmYxM2FiMTQ $3 \mathrm{OGM}_{3} \mathrm{MTFjYw}$

17. Valero-Rello A, Henares D, Acosta L, Jane M, Jordan I, Godoy P, et al. Validation and Implementation of a Diagnostic Algorithm for DNA Detection of Bordetella pertussis, B. parapertussis and B. holmesii in a Pediatric Referral Hospital in Barcelona, Spain. J Clin Microbiol. 2019;57(1):e01231-18. https://doi. org/10.1128/JCM.01231-18 PMID: 30404946

18. World Medical Association. World Medical Association Declaration of Helsinki: ethical principles for medical research involving human subjects. JAMA. 2013;310(20):2191-4. https:// doi.org/10.1001/jama.2013.281053 PMID: 24141714

19. De Greeff SC, Dekkers ALM, Teunis P, Rahamat-Langendoen JC, Mooi FR, De Melker HE. Seasonal patterns in time series of pertussis. Epidemiol Infect. 2009;137(10):1388-95. https://doi. org/10.1017/So950268809002489 PMID: 19327200

20. Wang Y, Xu C, Wang Z, Zhang S, Zhu Y, Yuan J. Time series modeling of pertussis incidence in China from 2004 to 2018 with a novel wavelet based SARIMA-NAR hybrid model. PLoS One. 2018;13(12):e0208404. https://doi.org/10.1371/journal. pone.0208404 PMID: 30586416

21. European Commission. Commission Implementing Decision (EU) $2018 / 945$ of 22 June 2018 on the communicable diseases and related special health issues to be covered by epidemiological surveillance as well as relevant case definitions (Text with EEA relevance). Official Journal of the European Union. Luxembourg: Publications Office of the European Union. 6.7.2018:L 170/1. Available from: https://eurlex.europa.eu/legal-content/EN/TXT/PDF/?uri=CELEX:32018Do 945\&from $=E N \#$ page $=32$

22. van der Maas NAT, Hoes J, Sanders EAM, de Melker HE. Severe underestimation of pertussis related hospitalizations and deaths in the Netherlands: A capture-recapture analysis. Vaccine. 2017;35(33):4162-6. https://doi.org/10.1016/j. vaccine.2017.06.037 PMID: 28651837

23. Crowcroft NS, Booy R, Harrison T, Spicer L, Britto J, Mok $Q$, et al. Severe and unrecognised: pertussis in UK infants. Arch Dis Child. 2003;88(9):802-6. https://doi.org/10.1136/ adc.88.9.802 PMID: 12937105
24. Godoy P, García-Cenoz M, Toledo D, Carmona G, Caylà JA, Alsedà $M$, et al. , Transmission of Pertussis in Households Working Group. Factors influencing the spread of pertussis in households: a prospective study, Catalonia and Navarre, Spain, 2012 to 2013. Euro Surveill. 2016;21(45):30393. https:// doi.org/10.2807/1560-7917.ES.2016.21.45.30393 PMID: 27918260

25. Skoff TH, Kenyon C, Cocoros N, Liko J, Miller L, Kudish K, et al. Sources of Infant Pertussis Infection in the United States. Pediatrics. 2015;136(4):635-41. https://doi.org/10.1542/ peds.2015-1120 PMID: 26347437

26. World Health Organization (WHO). Laboratory Manual for the diagnosis of whooping cough caused by bordetella pertussis/ bordetella parapertussis: update 2014. Geneva: WHO; 2014. Available from: https://apps.who.int/iris/handle/10665/127891

27. Pittet LF, Emonet S, Schrenzel J, Siegrist C-A, Posfay-Barbe KM. Bordetella holmesii: an under-recognised Bordetella species. Lancet Infect Dis. 2014;14(6):510-9. https://doi.org/10.1016/ S1473-3099(14)70021-0 PMID: 24721229

28. Liko J, Robison SG, Cieslak PR. Do Pertussis Vaccines Protect Against Bordetella parapertussis? Clin Infect Dis. 2017;64(12):1795-7. https://doi.org/10.1093/cid/cix221 PMID: 28369240

\section{License, supplementary material and copyright}

This is an open-access article distributed under the terms of the Creative Commons Attribution (CC BY 4.0) Licence. You may share and adapt the material, but must give appropriate credit to the source, provide a link to the licence and indicate if changes were made.

Any supplementary material referenced in the article can be found in the online version.

This article is copyright of the authors or their affiliated institutions, 2021. 